\title{
3 Research Square \\ Clinical Features of Pregnant Women and Neonates with COVID-19 in Wuhan: a paired comparison study
}

\section{Qin Xie}

Wuhan University Zhongnan Hospital

\section{Quan Gan}

women and children's hospital of hubei province

Jing Hu

women and children's hospital of hubei province

Dong Zhou

women and children's hospital of hubei province

\section{Shuhan Cai}

Wuhan University Zhongnan Hospital

\section{Lianjiu Su}

Wuhan University Zhongnan Hospital

\section{Yunxiang Li}

women and children's hospital of hubei province

\section{Yiming Li}

Wuhan University Zhongnan Hospital

Zhiyong Peng ( $\nabla$ zn001590@whu.edu.cn )

Wuhan University Zhongnan Hospital https://orcid.org/0000-0002-0849-5648

\section{Research article}

Keywords: COVID-19, Pregnant women, Neonates, Clinical features, Paired comparison study

Posted Date: June 12th, 2020

DOI: https://doi.org/10.21203/rs.3.rs-31339/v1

License: (c) (1) This work is licensed under a Creative Commons Attribution 4.0 International License.

Read Full License 


\section{Abstract \\ Background}

Our study aimed to find symptoms unique in pregnancy and to help the early diagnosis in pregnant women and to evaluate the impact of COVID-19 in postpartum women and their newborns.

\section{Methods}

Clinical data were reviewed and collected for 11 pregnant women with laboratory-confirmed COVID-19 who were consecutively admitted to Zhongnan Hospital of Wuhan University and Women and Children's Hospital of Hubei Province, from Jan 26 to Feb 26, 2020.

\section{Results}

All the confirmed women didn't have any exposure history and their early symptoms were mildly elevated temperate and fatigue. The chest CT scans of confirmed women can be atypical manifestations, such as bilateral pleural effusions and slightly increased densities. Eight of eleven confirmed women did not feel anything unusual until abnormalities were found on chest CT scans on admission screening test. All three groups had elevated white blood cell count, neutrophil count and lactate dehydrogenase, and reduced total protein.The infection did not increase the risk for premature delivery, premature rupture of membrane, or comorbidities in pregnancy.

\section{Conclusions}

Pregnant women were often asymptomatic and accidentally detected abnormalities on chest CT scan on admission which emphasize the importance of CT scan in prevalent areas of the COVID-19. Even after the laboratory confirmation, the manifestation of the CT scan could be atypical, which alerted the necessity of protection for healthcare workers. The COVID-19 did not increase the risk of complications in pregnant women and their neonates.

\section{Trial registration:}

This case series was approved by the institutional ethics board of Zhongnan Hospital of Wuhan University (No. 2020020) and Women and Children's Hospital of Hubei Province(NO. LW035).

\section{Introduction}


Coronavirus disease 2019 (COVID-19) is a highly infectious caused by severe acute respiratory syndrome coronavirus 2 (SARS-CoV-2).[1-3] The disease was first identified in Wuhan, Hubei province, China and has since spread globally causing the ongoing pandemic.[4-6] The World Health Organization (WHO) declared the 2019-20 coronavirus outbreak a Public Health Emergency of International Concern (PHEIC) on 30 January 2020, and a pandemic on 11 March 2020.[7] As of 2 April 2020, more than 937,100 cases of COVID-19 have been reported in over 200 countries and territories, resulting in approximately 47,266 deaths.[8] Common symptoms in adult patients include fever, cough, and shortness of breath. $[9,10]$ Other symptoms may include muscle pain, sputum production, diarrhea, sore throat, loss of smell, and abdominal pain.[11-13]

However, pregnant women and neonates are particularly susceptible to the disease because they are in a special state of immune suppression. The symptoms and outcomes in this special population are not well understood. Whether the symptoms in pregnant women with COVID-19 will demonstrate different symptoms other than the general population, whether the infection during pregnancy will influence the fetus, and whether infants born to mothers of confirmed COVID-19 will be more likely to develop neonates respiratory distress syndrome (NRDS) and have an increased risk for complications are questions that need prompt answer for better clinical diagnosis and management. Therefore, we conducted a two-center research hoping to find the answers. Women and Children's Hospital of Hubei Province is one of the largest centers for delivery of the province with around 30,000 deliveries per year. Zhongnan Hospital of Wuhan University is a tertiary hospital for COVID-19 patients. Both hospitals are centers for intensive care of pregnant women and children.

\section{Methods}

\section{Study Design and Participants}

Our study included 33 pregnant women admitted to Zhongnan Hospital of Wuhan University and Women and Children's Hospital of Hubei Province from January 26 to February 26, 2020. All the patients included were residents in Wuhan and were in their third trimester of pregnancy.

We identified eleven pregnant women with PCR-confirmed SARS-CoV-2 pneumonia in the confirmed group. A confirmed case of COVID-19 was defined as a positive result on high-throughput sequencing or real-time reverse-transcriptase-polymerase-chain-reaction (RT-PCR) assay of nasal and pharyngeal swab specimens. We then included 11 age-paired pregnant women suspicious of SARS-CoV-2 pneumonia in the suspected group. These patients had radiologic manifestations of COVID-19 pneumonia, and had at least one of the two following clinical symptoms: 1 . Fever and/or respiratory symptoms; 2 . Normal or decreased white blood cell count and reduced lymphocyte count in the early stages of onset, but were negative for RT-PCR test, despite of repeated tests. Radiologic manifestations on chest Computed tomography (CT) of COVID-19 pneumonia were defined as: multiple small patchy shadowing and interstitial changes in early stage, especially in the subpleural area; further developments into multiple ground glass opacities and bilateral infiltrates; pulmonary consolidation can be seen in severe cases; 
pleural effusions are rare. We randomly chose 11 healthy pregnant women in the normal group, matched by age, for each PCR-confirmed SARS-CoV-2 patient. These women had no symptoms or abnormalities on the screening chest CT. All the pregnant women were in third trimester when admitted and their neonates were also included in the study. Diagnosis of the NCP was based on the New Coronavirus Pneumonia Prevention and Control Program (5th edition) published by the National Health Commission of China.[14]

This study was reviewed and approved by the Medical Ethical Committee of both Zhongnan Hospital of Wuhan University and Women and Children's Hospital of Hubei Province.

\section{Data Collection}

The medical records of all the patients were extracted from the electronic medical systems by the research team of the Department of Critical Care Medicine of Zhongnan Hospital of Wuhan University and Women and Children's Hospital of Hubei Province.

Demographic data, medical history, exposure history, underlying comorbidities, signs and symptoms, laboratory findings, chest computed tomographic (CT) scans, delivery records, perinatal exams and treatment were obtained for all the pregnant women. Clinical manifestations, Apgar scores, throat swab, chest X-ray, laboratory findings and treatment were collected for all the neonates. Diagnosis of suspects of COVID-19 pneumonia was made by independent reviewing of chest CT scans by at least two investigators. Major disagreement between two reviewers was resolved by consultation with a third reviewer. The data were reviewed by a trained team of physicians, the missing data required a second check and confirmation with the coordinators of each hospital. Specimens (nasopharyngeal swabs for both mothers and neonates) were collected at multiple time points during the perinatal period for about 2 weeks and tested by RT-PCR for the presence of SARS-CoV-2, following WHO guidelines.[15-17]

\section{Statistical Analysis}

We assessed the association between SARS-CoV-2 virus infection and subsequent symptoms, laboratory findings and outcomes, by comparing the laboratory-confirmed pregnant women and neonates versus matched comparisons in the suspected group, and matched healthy pregnant women and neonates in the normal group.

Categorical variables were expressed as counts and percentages. The Fishers exact test was used to compare the differences between two groups. Continuous variables were expressed as medians and interquartile ranges. All continuous variables underwent Shapiro-Wilk test for check of normality in both pregnant women and neonates. T-test was used for comparison of 2 groups of continuous data with normal distribution. Otherwise, the Mann-Whitney $U$ test was used. Paired-samples test was applied in groups of pregnant women; however, two-independent-samples test was applied in groups of neonates because many neonates were not hospitalized for further examinations and the paired cohorts were not 
possible. For all variables, a two-sided $P$ value of $<0.05$ was considered statistically significant. Statistical analysis was performed with SPSS (mac0S 26.0 version).

\section{Results}

\section{Clinical features of pregnant women}

Our study identified 11 pregnant women with confirmed NCP (positive for both chest CT scans and RTPCR), 10 patients were categorized as nonsevere disease and 1 was severe disease. And they were matched by age with 11 pregnant women suspected of NCP (positive for chest CT scans but negative for RT-PCR), and 11 normal pregnant women (negative for both chest CT scans and RT-PCR). They were subdivided into the confirmed group, the suspected group, and the normal group, respectively.

The demographic and clinical characteristics of the patients are shown in Table 1. All the 33 women involved were residents of Wuhan and they were infected during their third trimester and had the delivery at the hospital. The median age of the patients was 30 years (interquartile range, 29 to 33) for all three groups. None of the women of the confirmed group and the normal group had any contact with patients suspicious of COVID-19 nor could they recall any. Three women of the suspected group had close contact with suspects, two of which were their husbands and one was her mother-in-law, however, none of these suspects was confirmed with COVID-19. 
Table 1

Clinical Characteristics of Three Groups of Pregnant Women

Characteristic
Confirmed group $(n=11)$
Confirmed group vs.

Suspected group

Suspected

group $(n=11)$
$P$ value
Confirmed group vs. Normal group

Normal $\quad P$ group $(n=\quad$ value 11)

Age

Median (IQR), yr $30.0(29.0-$ 33.0)

$30.0(29.0-$ 33.0)

1.00

$30.0(29.0-$ 33.0)

Distribution, $\mathrm{n} \%$

\begin{tabular}{llllll}
$25-29 \mathrm{yr}$ & $3(27.3)$ & $3(27.3)$ & 1.00 & $3(27.3)$ & 1.00 \\
$30-34 \mathrm{yr}$ & $7(63.6)$ & $7(63.6)$ & 1.00 & $7(63.6)$ & 1.00 \\
$>$ > 34 yr & $1(9.1)$ & $1(9.1)$ & 1.00 & $1(9.1)$ & 1.00 \\
$\begin{array}{l}\text { Gestational age at infection, } \\
\text { median (IQR), weeks }\end{array}$ & $\begin{array}{l}37.9(34.9- \\
40.4)\end{array}$ & $\begin{array}{l}36.7(35.1- \\
38.3)\end{array}$ & 0.29 & $38.1(37.4-$ & 0.97 \\
\hline
\end{tabular}

Exposure to source of

transmission within past 14

days

Living in Wuhan

Had contact with suspicious

patient, $\mathrm{n} \%$

$\begin{array}{lllll}11(100.0) & 11(100.0) & 1.00 & 11(100.0) & 1.00 \\ 0(0.0) & 3(27.3) & 0.21 & 0(0.0) & \text { NA }\end{array}$

Fever

\begin{tabular}{|c|c|c|c|c|c|}
\hline Patients, n\% & $5(45.5)$ & $8(72.7)$ & 0.39 & $0(0.0)$ & 0.04 \\
\hline $\begin{array}{l}\text { Peak temperature }{ }^{\square} \text {, median } \\
\text { (IQR), }{ }^{\circ} \mathrm{C}\end{array}$ & $\begin{array}{l}37.0(36.7- \\
38.0)\end{array}$ & $\begin{array}{l}37.6(36.5- \\
37.8)\end{array}$ & 0.63 & $\begin{array}{l}36.7(36.5- \\
36.9)\end{array}$ & 0.02 \\
\hline $\begin{array}{l}\text { Distribution of temperature, } n \% \text {, } \\
{ }^{\circ} \mathrm{C}\end{array}$ & & & 1.00 & & 0.04 \\
\hline$<37.5^{\circ} \mathrm{C}$ & $6(54.5)$ & $4(36.4)$ & & $11(100.0)$ & \\
\hline
\end{tabular}

$\mathrm{IQR}=$ interquartile-range. $\mathrm{PIH}=$ pregnancy-induced hypertension. $\mathrm{GDM}=$ gestational diabetes mellitus. $\mathrm{CSP}=$ cesarean scar pregnancy. $\mathrm{PROM}=$ premature rupture of membrane. $\mathrm{C}$-section = cesareansection. IVF = in vitro fertilization.

Q Peak temperature noted within 14 days following the pneumonia.

$\nabla$ Hypertension that pre-existed before this pregnancy.

Q Included in this category are coronary heart disease, chronic obstructive pulmonary disease, cerebrovascular disease, chronic renal disease, infectious disease, malignancy, and immunodeficiency. 


\begin{tabular}{|c|c|c|c|c|c|}
\hline \multirow{2}{*}{$\begin{array}{l}\text { Characteristic } \\
37.5-38.0^{\circ} \mathrm{C}\end{array}$} & \multirow{2}{*}{$\begin{array}{l}\text { Confirmed } \\
\text { group }(n=11) \\
3(27.3)\end{array}$} & \multicolumn{2}{|c|}{$\begin{array}{l}\text { Confirmed group vs. } \\
\text { Suspected group }\end{array}$} & \multicolumn{2}{|c|}{$\begin{array}{l}\text { Confirmed group vs. } \\
\text { Normal group }\end{array}$} \\
\hline & & $6(54.5)$ & & $0(0.0)$ & \\
\hline $38.1-39.0^{\circ} \mathrm{C}$ & $1(9.1)$ & $1(9.1)$ & & $0(0.0)$ & \\
\hline$>39.0^{\circ} \mathrm{C}$ & $1(9.1)$ & $0(0.0)$ & & $0(0.0)$ & \\
\hline \multicolumn{6}{|l|}{ Symptoms, n\% } \\
\hline Cough & $2(18.2)$ & $3(27.3)$ & 1.00 & $0(0.0)$ & 0.48 \\
\hline Fatigue & $5(45.5)$ & $0(0.0)$ & 0.04 & $0(0.0)$ & 0.04 \\
\hline Sore throat & $1(9.1)$ & $1(9.1)$ & 1.00 & $0(0.0)$ & 1.00 \\
\hline Dyspnea & $0(0.0)$ & $1(9.1)$ & 1.00 & $0(0.0)$ & NA \\
\hline Headache & $0(0.0)$ & $1(9.1)$ & 1.00 & $0(0.0)$ & NA \\
\hline Myalgia or arthralgia & $0(0.0)$ & $0(0.0)$ & NA & $0(0.0)$ & NA \\
\hline Palpitation & $0(0.0)$ & $0(0.0)$ & NA & $0(0.0)$ & NA \\
\hline Diarrhea & $0(0.0)$ & $0(0.0)$ & NA & $0(0.0)$ & NA \\
\hline \multicolumn{6}{|c|}{ Coexisting disorders, n\% } \\
\hline Any & $5(45.5)$ & $4(36.4)$ & 1.00 & $9(81.8)$ & 0.18 \\
\hline Hypertension ${ }^{\square}$ & $1(9.1)$ & $0(0.0)$ & 1.00 & $0(0.0)$ & 1.00 \\
\hline $\mathrm{PIH}$ & $2(18.2)$ & $2(18.2)$ & 1.00 & $2(18.2)$ & 1.00 \\
\hline GMD & $1(9.1)$ & $2(18.2)$ & 1.00 & $2(18.2)$ & 1.00 \\
\hline CSP & $3(27.3)$ & $1(9.1)$ & 0.59 & $7(63.6)$ & 0.20 \\
\hline PROM & $2(18.2)$ & $1(9.1)$ & 1.00 & $2(18.2)$ & 1.00 \\
\hline Hypothyroidism & $2(18.2)$ & $0(0.0)$ & 0.05 & $0(0.0)$ & 0.05 \\
\hline Other & $0(0.0)$ & $0(0.0)$ & NA & $0(0.0)$ & NA \\
\hline
\end{tabular}

IQR = interquartile-range. $\mathrm{PIH}=$ pregnancy-induced hypertension. $\mathrm{GDM}=$ gestational diabetes mellitus. $\mathrm{CSP}=$ cesarean scar pregnancy. $\mathrm{PROM}=$ premature rupture of membrane. $\mathrm{C}$-section = cesareansection. IVF = in vitro fertilization.

Q Peak temperature noted within 14 days following the pneumonia.

$\nabla$ Hypertension that pre-existed before this pregnancy.

Q Included in this category are coronary heart disease, chronic obstructive pulmonary disease, cerebrovascular disease, chronic renal disease, infectious disease, malignancy, and immunodeficiency. 


\begin{tabular}{|c|c|c|c|c|c|}
\hline \multirow{2}{*}{$\begin{array}{l}\text { Characteristic } \\
\text { Past medical history, n\% }\end{array}$} & \multirow[t]{2}{*}{$\begin{array}{l}\text { Confirmed } \\
\text { group }(n=11)\end{array}$} & \multicolumn{2}{|c|}{$\begin{array}{l}\text { Confirmed group vs. } \\
\text { Suspected group }\end{array}$} & \multicolumn{2}{|c|}{$\begin{array}{l}\text { Confirmed group vs. } \\
\text { Normal group }\end{array}$} \\
\hline & & & & & \\
\hline Surgeries & $5(45.5)$ & $4(36.4)$ & 1.00 & $8(72.7)$ & 0.39 \\
\hline Smoking or drinking & $0(0.0)$ & $0(0.0)$ & NA & $0(0.0)$ & NA \\
\hline Transfusion & $0(0.0)$ & $0(0.0)$ & NA & $0(0.0)$ & NA \\
\hline \multicolumn{6}{|l|}{ Delivery information } \\
\hline C-section, n\% & $9(81.8)$ & $11(100.0)$ & 0.48 & $8(72.7)$ & 1.00 \\
\hline Premature delivery & $4(30.8)$ & $3(27.3)$ & 1.00 & $3(25.0)$ & 1.00 \\
\hline IVF, n\% & $2(18.2)$ & $2(18.2)$ & 1.00 & $0(0.0)$ & 0.48 \\
\hline Twin pregnancy, n\% & $2(18.2)$ & $0(0.0)$ & 0.48 & $1(9.1)$ & 1.00 \\
\hline Gestation times, median (IQR) & $1(1-3)$ & $1(1-2)$ & 0.59 & $2(2-3)$ & 0.20 \\
\hline Parturition times, median (IQR) & $0(0-1)$ & $0(0-1)$ & 1.00 & $1(0-1)$ & 0.10 \\
\hline $\begin{array}{l}\text { Abdominal girth, median (IQR), } \\
\mathrm{cm}\end{array}$ & $\begin{array}{l}105.0(99.0- \\
108.0)\end{array}$ & $\begin{array}{l}105.0(96.0- \\
106.0)\end{array}$ & 0.48 & $\begin{array}{l}100.0(92.0- \\
105.0)\end{array}$ & 0.30 \\
\hline Fundal height, median (IQR), cm & $\begin{array}{l}35.0(33.0- \\
36.0)\end{array}$ & $\begin{array}{l}35.0(33.0- \\
35.0)\end{array}$ & 0.62 & $\begin{array}{l}35.0(33.0- \\
36.0)\end{array}$ & 0.78 \\
\hline $\begin{array}{l}\text { Fetal heart rate, median (IQR), } \\
\text { bmp }\end{array}$ & $\begin{array}{l}146.0(140.0- \\
148.0)\end{array}$ & $\begin{array}{l}140.0(140.0- \\
145.0)\end{array}$ & 0.02 & $\begin{array}{l}145.0 \\
(142.0- \\
148.0)\end{array}$ & 0.87 \\
\hline \multicolumn{6}{|c|}{$\begin{array}{l}\text { IQR = interquartile-range. } \mathrm{PIH}=\text { pregnancy-induced hypertension. } \mathrm{GDM}=\text { gestational diabetes mellitus. } \\
\mathrm{CSP}=\text { cesarean scar pregnancy. } \mathrm{PROM}=\text { premature rupture of membrane. C-section = cesarean- } \\
\text { section. IVF = in vitro fertilization. }\end{array}$} \\
\hline \multicolumn{6}{|c|}{$\otimes$ Peak temperature noted within 14 days following the pneumonia. } \\
\hline \multicolumn{6}{|c|}{$\otimes$ Hypertension that pre-existed before this pregnancy. } \\
\hline $\begin{array}{l}Q \text { Included in this category are cor } \\
\text { cerebrovascular disease, chronic } \\
\text { immunodeficiency. }\end{array}$ & $\begin{array}{l}\text { ary heart dise } \\
\text { al disease, in }\end{array}$ & $\begin{array}{l}\text { chronic obstr } \\
\text { ous disease, I }\end{array}$ & $\begin{array}{l}\text { ve pu } \\
\text { gnan }\end{array}$ & $\begin{array}{l}\text { nary disease, } \\
\text { ind }\end{array}$ & \\
\hline
\end{tabular}

Fever was present in $45.5 \%$ of the confirmed pregnant women and in $72.7 \%$ of the suspected women. The median peak temperatures in both groups were moderately elevated (less than $38^{\circ} \mathrm{C}$ ), only one patient in the confirmed group had a high fever $\left(>39^{\circ} \mathrm{C}\right)$. However, there were no significant differences of peak temperature or the numbers of different temperature ranks between two groups. Other symptoms of confirmed group and suspected group included cough ( $18.2 \%$ vs. $27.3 \%, p=1.00)$, fatigue $(45.5 \%$ vs $0.0 \%$, $p=0.04)$, sore throat $(9.1 \%$ vs. $9.1 \%, p=1.00)$, dyspnea and headache $(0.0 \%$ vs. $9.1 \%, p=1.00)$. Except for fatigue $(p=0.04)$, none of the symptoms above in the confirmed group had statistical significance 
comparing to the suspected group and the normal group. Comparing to the normal group, the distinguishable symptoms of pregnant women with COVID-19 was mildly elevated body temperature and fatigue $(p<0.05)$.

Only 1 out of 33 pregnant women had hypertension (3.0\%) as preexisting disease. Overall, coexisting diseases during pregnancy included pregnancy-induced hypertension $(\mathrm{PIH}, 18.2 \%)$, gestational diabetes mellitus (GDM, 15.2\%), cesarean scar pregnancy (CSP, 33.3\%), premature rupture of membrane (PROM, $15.2 \%)$, and hypothyroidism (6.1\%). Of all the patients, 17 (51.5\%) had surgeries, of which 10 (58.8\%) were $\mathrm{C}$-section delivery, but no one ever had a smoking and drinking history or had a transfusion. None of the comorbidities nor past medical history mentioned above was statistically significant among these three groups. The COVID-19 didn't induce the risk for PROM, premature delivery or comorbidities, like GMD, PIH in pregnant women.

\section{Vital Signs, Radiologic And Laboratory Findings Of Pregnant Women}

Table 2 shows the vital signs on admission and the radiologic and laboratory findings during hospitalization. The vital signs of pregnant women on admission were all stable. Comparing to the suspected group, the confirmed group had a higher median heart rate (92 bpm vs. $80 \mathrm{bpm}, p=0.03$ ). And comparing to the normal group, the confirmed group had a higher median diastolic blood pressure (DBP, $87 \mathrm{mmHg}$ vs. $75 \mathrm{mmHg}, \mathrm{p}=0.04$ ) and a higher median mean arterial pressure (MAP, $101 \mathrm{mmHg}$ vs. $88 \mathrm{mmHg}, p=0.04)$. None of the patients had hypoxemia. 
Table 2

Vital Signs, Radiologic and Laboratory Findings

\section{Variable}

Confirmed group $(n=11)$
Confirmed group vs.

Suspected group

Suspected group $(n=11)$
$36.5(36.5-$

37.0)

Heart rate, bpm

Systolic pressure, $\mathrm{mmHg}$

Diastolic pressure, $\mathrm{mmHg}$

Mean arterial pressure,

$\mathrm{mmHg}$

Hypoxemia, $n \%$

RT-PCR positive, $\mathrm{n} \%$

Radiologic findings, $\mathrm{n} \%$

Any

Ground-glass opacity

Bilateral infiltrates

Abnormality ranges

$0 \sim 10 \%$

$10 \sim 25 \%$

$25 \sim 50 \%$

Laboratory findings,

median (IQR)

Complete Blood Count

Red blood cell count, $\times 10^{12} / \mathrm{L}$

Hemoglobin, g/L

Temperature, ${ }^{\circ} \mathrm{C}$

$87(78-94)$

$0(0.0)$

$11(100.0)$

$11(100.0)$

$10(90.9)$

7 (63.6)
$36.5(36.4-37.6) \quad 0.38$

$P \quad$ Normal group Value $\quad(n=11)$
Confirmed group vs.

Normal group

$P$

Value

admission

median (IQR)

$92(80-100) \quad 80(78-80)$

$128(121-148)$
$101(93-112)$

$87(78-97)$

$99(94-110)$

$0(0.0)$

$0(0.0)$

$0.00 \quad 0(0.0)$

NA $\quad 0(0.0)$

NA

$0.03 \quad 88(78-105) \quad 0.76$

$0.37 \quad 120(107-$ 125)

0.05

$$
\text { 125) }
$$

$0.93 \quad 75(70-82)$

$0.66 \quad 88(83-100) \quad 0.04$

$0.66 \quad 88(83-100) \quad \mathbf{0 . 0 4}$

0.00 


\begin{tabular}{|c|c|c|c|c|c|}
\hline \multirow{2}{*}{$\begin{array}{l}\text { Variable } \\
\text { White blood cell count, } \\
\times 10^{9} / \mathrm{L}\end{array}$} & \multirow{2}{*}{$\begin{array}{l}\begin{array}{l}\text { Confirmed } \\
\text { group }(n=11)\end{array} \\
\uparrow 9.8(8.9-10.2)\end{array}$} & \multicolumn{2}{|c|}{$\begin{array}{l}\text { Confirmed group vs. } \\
\text { Suspected group }\end{array}$} & \multicolumn{2}{|c|}{$\begin{array}{l}\text { Confirmed group vs. } \\
\text { Normal group }\end{array}$} \\
\hline & & $\uparrow 10.6(7.8-14.2)$ & 0.93 & $\begin{array}{l}\uparrow 10.4(7.8- \\
11.1)\end{array}$ & 0.86 \\
\hline Neutrophil count, $\times 10^{9} / \mathrm{L}$ & $\uparrow 7.5(6.8-9.4)$ & $\uparrow 8.2(5.7-11.7)$ & 0.93 & $\uparrow 7.7(5.5-8.5)$ & 0.48 \\
\hline $\begin{array}{l}\text { Lymphocyte count, } \\
\times 10^{9} / \mathrm{L}\end{array}$ & $1.2(0.7-1.6)$ & $1.5(0.9-2.0)$ & 0.16 & $1.9(1.4-2.2)$ & 0.01 \\
\hline Platelet count, $\times 10^{9} / \mathrm{L}$ & $203(183-238)$ & $234(173-239)$ & 0.93 & $\begin{array}{l}210(184- \\
271)\end{array}$ & 0.40 \\
\hline \multicolumn{6}{|l|}{ Liver function tests } \\
\hline Prothrombin time, s & $10.3(9.9-10.7)$ & $10.8(10.3-11.2)$ & 0.29 & $\begin{array}{l}10.1(9.7- \\
10.8)\end{array}$ & 0.91 \\
\hline $\begin{array}{l}\text { International normalized } \\
\text { ratio }\end{array}$ & $\begin{array}{l}0.96(0.92- \\
0.98)\end{array}$ & $0.99(0.94-1.03)$ & 0.69 & $\begin{array}{l}0.94(0.90- \\
1.00)\end{array}$ & 0.82 \\
\hline $\begin{array}{l}\text { Alanine } \\
\text { aminotransferase, U/L }\end{array}$ & $14.9(9.1-18.2)$ & $27.0(9.0-69.0)$ & 0.16 & $8.1(7.2-16.5)$ & 0.18 \\
\hline $\begin{array}{l}\text { Aspartate } \\
\text { aminotransferase, U/L }\end{array}$ & $23.7(19.0-30.1)$ & $29.0(19.0-36.0)$ & 0.21 & $\begin{array}{l}16.9(13.0- \\
22.1)\end{array}$ & 0.06 \\
\hline Total bilirubin, $\mu \mathrm{mol} / \mathrm{L}$ & $8.2(5.1-9.4)$ & $9.3(8.4-11.1)$ & 0.23 & $8.4(5.7-9.5)$ & 0.87 \\
\hline Total protein, $\mathrm{g} / \mathrm{L}$ & $\begin{array}{l}\text { J63.4 (59.0- } \\
66.8)\end{array}$ & $\begin{array}{l}\downarrow 59.5(54.0- \\
70.6)\end{array}$ & 0.70 & $\begin{array}{l}\downarrow 65.8(64.8- \\
69.8)\end{array}$ & 0.09 \\
\hline Albumin, $\mathrm{g} / \mathrm{L}$ & $\begin{array}{l}\downarrow 32.9(30.4- \\
34.6)\end{array}$ & $\begin{array}{l}\downarrow 31.8(28.5- \\
38.7)\end{array}$ & 0.76 & $\begin{array}{l}36.5(34.8- \\
38.3)\end{array}$ & 0.02 \\
\hline \multicolumn{6}{|l|}{ Other findings } \\
\hline $\begin{array}{l}\text { Blood urea nitrogen, } \\
\mathrm{mmol} / \mathrm{L}\end{array}$ & $3.1(2.4-3.4)$ & $\downarrow 2.7(2.5-4.1)$ & 0.93 & $3.5(2.9-3.9)$ & 0.37 \\
\hline Creatinine, $\mu \mathrm{mol} / \mathrm{L}$ & $\begin{array}{l}53.4(46.2- \\
58.9)\end{array}$ & $54.8(46.0-65.8)$ & 0.72 & $\begin{array}{l}48.0(42.6- \\
57.9)\end{array}$ & 0.33 \\
\hline Creatinine kinase, $\mathrm{U} / \mathrm{L}$ & $\begin{array}{l}93.2(54.6- \\
116.0)\end{array}$ & $\begin{array}{l}65.4(31.0- \\
138.0)\end{array}$ & 0.48 & $\begin{array}{l}68.6(39.3- \\
80.2)\end{array}$ & 0.06 \\
\hline $\begin{array}{l}\text { Lactate dehydrogenase, } \\
\text { U/L }\end{array}$ & $\begin{array}{l}241.0(195.5- \\
253.2)\end{array}$ & $\begin{array}{l}193.0(168.0- \\
353.7)\end{array}$ & 1.00 & $\begin{array}{l}183.9(153.0- \\
201.8)\end{array}$ & 0.00 \\
\hline D-dimer, $\mu \mathrm{g} / \mathrm{mL}$ & $\begin{array}{l}\uparrow 1.69(1.13- \\
4.77)\end{array}$ & $\begin{array}{l}\uparrow 2.43(0.62- \\
3.38)\end{array}$ & 0.48 & $\begin{array}{l}\uparrow 1.71(1.28- \\
2.31)\end{array}$ & 0.33 \\
\hline $\begin{array}{l}\text { Electrocardiograph } \\
\text { abnormalities, n\% }\end{array}$ & $0(0.0)$ & $0(0.0)$ & NA & $0(0.0)$ & NA \\
\hline
\end{tabular}


All patients in the confirmed group had at least once positive results of RT-PCR for detection of SARSCoV-2 virus, the other groups were negative for the result. All the pregnant women had a routine screening of chest CT scan on admission (Fig. 1), the major chest CT scan findings in the confirmed group and the suspected group were ground-glass opacity $(90.9 \%, 100.0 \%)$ and bilateral infiltrates $(63.6 \%, 63.6 \%)$. Abnormality mainly ranged from $0-25 \%$ of the lungs. The CT scan of the confirmed patients could be atypical manifestations, such as pleural effusions and increased densities. Eight of eleven (72.7\%) confirmed women did not feel anything unusual until abnormalities were found on chest CT scans on admission screening test.

All three groups had elevated white blood cell count, neutrophil count and lactate dehydrogenase, and reduced total protein, other liver function tests and renal function tests were normal. Comparing to the normal group, women in the confirmed group had significantly reduced hemoglobin, lymphocyte, albumin, and increased lactate dehydrogenase $(p<0.05)$. No statistical differences were found between the confirmed group and the suspected group.

\section{Treatment After Delivery, Complications And Outcomes Of Pregnant Women}

More than $80 \%$ women were received antibiotic therapy postoperatively (Table 3). Only patients in the confirmed group and the suspected group were treated with nasal cannula oxygen support, interferon inhalation and antiviral therapy. Patients in the confirmed group had a higher proportion of oxygen support and interferon inhalation than the suspected group (54.5\% vs. 9,1\%). None of the patients needed mechanical ventilation or systemic glucocorticoids. During hospital admission, none of the patients developed any complications, such as ARDS, AKI, arrhythmia, shock. At the end of the follow-up, all the patients of the normal group were discharged from the hospital, and $18.2 \%, 36.4 \%$ of the patients in the confirmed and suspected group were discharged. A majority of the patients $(72.7 \%)$ of the confirmed group were transferred to hospitals specialized for COVID-19. None of the patients was died. 
Table 3

Treatment after delivery, complications and outcomes of pregnant women

\begin{tabular}{|c|c|c|c|c|c|}
\hline \multirow[t]{2}{*}{ Variable } & \multirow[t]{2}{*}{$\begin{array}{l}\text { Confirmed group } \\
(n=11)\end{array}$} & \multicolumn{2}{|c|}{$\begin{array}{l}\text { Confirmed group vs. } \\
\text { Suspected group }\end{array}$} & \multicolumn{2}{|c|}{$\begin{array}{l}\text { Confirmed group vs. } \\
\text { Normal group }\end{array}$} \\
\hline & & $\begin{array}{l}\text { Suspected group } \\
(n=11)\end{array}$ & $\begin{array}{l}\mathrm{P} \\
\text { value }\end{array}$ & $\begin{array}{l}\text { Normal group ( } \mathrm{n} \\
=11 \text { ) }\end{array}$ & $\begin{array}{l}P \\
\text { value }\end{array}$ \\
\hline \multicolumn{6}{|l|}{$\begin{array}{l}\text { Treatment after } \\
\text { delivery, } \mathrm{n} \%\end{array}$} \\
\hline Oxygen support* & $6(54.5)$ & $1(9.1)$ & 0.06 & $0(0.0)$ & 0.01 \\
\hline $\begin{array}{l}\text { Interferon } \\
\text { inhalation }\end{array}$ & $6(54.5)$ & $1(9.1)$ & 0.06 & $0(0.0)$ & 0.01 \\
\hline Antiviral therapy & $2(18.2)$ & $1(9.1)$ & 1.00 & $0(0.0)$ & 0.48 \\
\hline Antibiotic therapy & $10(90.9)$ & $9(81.8)$ & 1.00 & $11(100.0)$ & 1.00 \\
\hline $\begin{array}{l}\text { Use of } \\
\text { corticosteroid }\end{array}$ & $0(0.0)$ & $0(0.0)$ & NA & $0(0.0)$ & NA \\
\hline Complications, n\% & $0(0.0)$ & $0(0.0)$ & NA & $0(0.0)$ & NA \\
\hline \multicolumn{6}{|l|}{ Outcomes, n\% } \\
\hline $\begin{array}{l}\text { Discharge from } \\
\text { hospital }\end{array}$ & $2(18.2)$ & $4(36.4)$ & 0.64 & $11(100.0)$ & 0.00 \\
\hline $\begin{array}{l}\text { Transfer to other } \\
\text { hospital }\end{array}$ & $8(72.7)$ & $4(36.4)$ & 0.20 & $0(0.0)$ & 0.00 \\
\hline Hospitalization & $1(9.1)$ & $3(27.3)$ & 0.59 & $0(0.0)$ & 1.00 \\
\hline Death & $0(0.0)$ & $0(0.0)$ & NA & $0(0.0)$ & NA \\
\hline
\end{tabular}

\section{Clinical Features Of Neonates}

Among the neonates born to mothers with confirmed COVID-19, there were two pairs of twins, making altogether 13 newborns; 8 of them were males, 12 were born by C-section delivery, 4 of them were premature infants, and 3 were low birth weight infants (Table 4). Neonates born to mothers of the suspected group and the normal group shared similar distribution features, except for that there were only two females in the normal group $(p=0.04)$. There were no twins born in the suspected group $(n=11)$ and a pair of twins born in the normal group $(n=12)$. Vital signs, Apgar scores, length, weight at birth were within normal ranges for all infants of each group. Different symptoms were observed for infants of three groups during their hospitalization, such as fever, dyspnea, lethargy, moaning, feeding intolerance, and cyanosis; however, these symptoms were not statistically different among different groups. 
Table 4

Clinical features of neonates born to mothers of three groups

Variable
Confirmed

group $(n=13)$
Confirmed group vs.

Suspected group

Suspected

group $(n=11)$
$P$

Normal group

$(n=12)$
Confirmed group

vs. Normal group

Clinical characteristics

Baseline information, $n \%$

$\begin{array}{llllll}\text { Male sex } & 8(61.5) & 6(54.5) & 1.00 & 2(16.7) & 0.04 \\ \text { C-section } & 12(92.3) & 11(100.0) & 1.00 & 9(75.0) & 0.32 \\ \begin{array}{llll}\text { Low birth weight }(< \\ 2500 \mathrm{~g}), \mathrm{n} \%\end{array} & 3(23.1) & 1(9.1) & 0.60 & 3(25.0) & 1.00\end{array}$

Information on birth,

median (IQR)

Gestational age, weeks

38.0 (34.9-

38.3 (36.3-

39.4)

0.89

38.1 (35.9-

0.89

39.8)

$49.0(48.0-$

$50.0)$

0.88

38.8)

value

Length, $\mathrm{cm}$

$50.0(46.5-$

50.0)

2810 (2670-

3150)

0.51

2950 (2480-

3500)

36.0 (35.9-

36.0)

$0.0136 .4(35.9-$

36.5)

$50.0(48.3-$

0.64

50.8)

Temperature, ${ }^{\circ} \mathrm{C}$

$36.2(36.5)$
$36.5)$

$40(40-40)$

$0.2640(40-48)$

1.00

Respiratory rate, bmp

40 (40-49)

130 (130-

130)

$\mathbf{0 . 0 4} 140(140-152)$

0.03

Heart rate, bpm

140 (130-

143)

48.3 (47.7-

48.5)

$48.3(44.7-$

51.3)

$0.52 \quad 41(41-41)$

0.00

$\mathrm{mmHg}$

Apgar score $<8, n \%$

$1 \mathrm{~min}$

$0(0.0)$

$1(9.1)$

$0.46 \quad 0(0.0)$

NA

C-section $=$ cesarean-section. IVF = in vitro fertilization

QThroat swab for SARS-CoV-2 virus were not done in all the neonates born to mother of the normal group.

Q Chest X-ray were not taken in $5(38.5 \%), 3(27.3 \%), 3(25.0 \%)$ neonates born to mothers of the confirmed group, the clinical diagnosis group, and the normal group, respectively.

Q Laboratory tests were not tested in 5 (38.5\%), 1 (9.1\%), 8 (66.7\%) neonates born to mothers of the confirmed group, the clinical diagnosis group, and the normal group, respectively. 


\begin{tabular}{|c|c|c|c|c|c|}
\hline \multirow{2}{*}{$\begin{array}{l}\text { Variable } \\
5 \mathrm{~min}\end{array}$} & \multirow{2}{*}{$\begin{array}{l}\text { Confirmed } \\
\text { group }(n=13) \\
0(0.0)\end{array}$} & \multicolumn{2}{|c|}{$\begin{array}{l}\text { Confirmed group vs. } \\
\text { Suspected group }\end{array}$} & \multicolumn{2}{|c|}{$\begin{array}{l}\text { Confirmed group } \\
\text { vs. Normal group }\end{array}$} \\
\hline & & $0(0.0)$ & NA & $0(0.0)$ & NA \\
\hline \multicolumn{6}{|l|}{ Symptoms, n\% } \\
\hline Fever & $1(7.7)$ & $0(0.0)$ & 1.00 & $3(25.0)$ & 0.32 \\
\hline Dyspnea & $3(23.1)$ & $1(9.1)$ & 0.60 & $1(8.3)$ & 0.59 \\
\hline Lethargy & $5(38.5)$ & $3(27.3)$ & 0.68 & $1(8.3)$ & 0.16 \\
\hline Moaning & $3(23.1)$ & $3(27.3)$ & 1.00 & $1(8.3)$ & 0.59 \\
\hline Feeding intolerance & $4(30.8)$ & $2(18.2)$ & 0.65 & $2(16.7)$ & 0.65 \\
\hline Cyanosis & $4(30.8)$ & $3(27.3)$ & 1.00 & $1(8.3)$ & 0.32 \\
\hline $\begin{array}{l}\text { Throat swab positive for } \\
\text { SARS-CoV- } 2^{\square}, n \%\end{array}$ & $0(0.0)$ & $0(0.0)$ & NA & NA & NA \\
\hline $\begin{array}{l}\text { Chest X-ray } \\
\text { abnormalities , no./total } \\
\text { no. (\%) }\end{array}$ & $2 / 8(25.0)$ & $3 / 8(37.5)$ & 0.63 & $2 / 9(22.2)$ & 1.00 \\
\hline \multicolumn{6}{|l|}{$\begin{array}{l}\text { Laboratory findings } \\
\text { median (IQR) }\end{array}$} \\
\hline $\begin{array}{l}\text { Red blood cell count, } \\
\times 10^{12} / \mathrm{L}\end{array}$ & $\downarrow 4.3(4.2-4.7)$ & $\downarrow 4.3(4.0-4.5)$ & 0.69 & $4.8(3.9-5.2)$ & 0.50 \\
\hline Hemoglobin, $\mathrm{g} / \mathrm{L}$ & $\begin{array}{l}166(158- \\
182)\end{array}$ & $\begin{array}{l}158(149- \\
188)\end{array}$ & 0.66 & $180(153-198)$ & 0.37 \\
\hline $\begin{array}{l}\text { White blood cell count, } \\
\times 10^{9} / \mathrm{L}\end{array}$ & $\begin{array}{l}10.5(8.2- \\
11.7)\end{array}$ & $\begin{array}{l}13.2(10.3- \\
18.9)\end{array}$ & 0.23 & $\begin{array}{l}15.3(12.2- \\
16.9)\end{array}$ & 0.06 \\
\hline Neutrophil count, $\times 10^{9} / \mathrm{L}$ & $5.3(4.7-6.8)$ & $8.4(6.0-12.0)$ & 0.04 & $7.5(4.8-11.7)$ & 0.40 \\
\hline $\begin{array}{l}\text { Lymphocyte count, } \\
\times 10^{9} / \mathrm{L}\end{array}$ & $3.5(3.1-4.4)$ & $2.6(2.1-3.8)$ & 0.13 & $5.4(3.9-6.9)$ & 0.03 \\
\hline
\end{tabular}

C-section = cesarean-section. IVF = in vitro fertilization .

$\otimes$ Throat swab for SARS-CoV-2 virus were not done in all the neonates born to mother of the normal group.

$\nabla$ Chest X-ray were not taken in $5(38.5 \%), 3(27.3 \%), 3(25.0 \%)$ neonates born to mothers of the confirmed group, the clinical diagnosis group, and the normal group, respectively.

$\nabla$ Laboratory tests were not tested in 5 (38.5\%), 1 (9.1\%), 8 (66.7\%) neonates born to mothers of the confirmed group, the clinical diagnosis group, and the normal group, respectively. 


\begin{tabular}{|c|c|c|c|c|c|}
\hline \multirow{2}{*}{$\begin{array}{l}\text { Variable } \\
\text { Platelet count, } \times 10^{9} / \mathrm{L}\end{array}$} & \multirow{2}{*}{$\begin{array}{l}\text { Confirmed } \\
\text { group }(n=13) \\
289(241- \\
335)\end{array}$} & \multicolumn{2}{|c|}{$\begin{array}{l}\text { Confirmed group vs. } \\
\text { Suspected group }\end{array}$} & \multicolumn{2}{|c|}{$\begin{array}{l}\text { Confirmed group } \\
\text { vs. Normal group }\end{array}$} \\
\hline & & $\begin{array}{l}201(173- \\
271)\end{array}$ & 0.10 & $317(275-368)$ & 0.41 \\
\hline $\begin{array}{l}\text { Alanine aminotransferase, } \\
\mathrm{U} / \mathrm{L}\end{array}$ & $\begin{array}{l}19.2(6.9- \\
12.5)\end{array}$ & $\begin{array}{l}\downarrow 11.0(8.8- \\
14.5)\end{array}$ & 0.26 & $\downarrow 7.3(6.6-13.7)$ & 0.55 \\
\hline $\begin{array}{l}\text { Aspartate } \\
\text { aminotransferase, U/L }\end{array}$ & $\begin{array}{l}50.8(39.0- \\
62.3)\end{array}$ & $\begin{array}{l}50.0(42.3- \\
73.8)\end{array}$ & 0.72 & $\begin{array}{l}39.7(30.9- \\
67.0)\end{array}$ & 0.59 \\
\hline Total bilirubin, $\mu \mathrm{mol} / \mathrm{L}$ & $\begin{array}{l}31.9(29.8- \\
38.5)\end{array}$ & $\begin{array}{l}72.3(41.8- \\
99.2)\end{array}$ & 0.01 & $33.7(31.6-2.6)$ & 0.74 \\
\hline Total protein, g/L & $\begin{array}{l}49.0(45.8- \\
61.5)\end{array}$ & $\begin{array}{l}50.8(46.9- \\
54.9)\end{array}$ & 0.92 & $\begin{array}{l}\downarrow 42.9(35.7- \\
53.9)\end{array}$ & 0.21 \\
\hline Albumin, $g / L$ & $\begin{array}{l}32.3(30.6- \\
36.1)\end{array}$ & $\begin{array}{l}33.0(31.9- \\
35.4)\end{array}$ & 0.96 & $\begin{array}{l}30.0(26.0- \\
35.0)\end{array}$ & 0.30 \\
\hline $\begin{array}{l}\text { Blood urea nitrogen, } \\
\mathrm{mmol} / \mathrm{L}\end{array}$ & $3.2(2.6-5.2)$ & $\downarrow 2.5(1.6-4.1)$ & 0.29 & $3.1(2.3-4.0)$ & 0.50 \\
\hline Creatinine, $\mu \mathrm{mol} / \mathrm{L}$ & $\begin{array}{l}36.5(16.6- \\
60.2)\end{array}$ & $\begin{array}{l}59.6(47.9- \\
73.7)\end{array}$ & 0.05 & $\begin{array}{l}\downarrow 15.6(11.5- \\
21.6)\end{array}$ & 0.32 \\
\hline Lactate, $\mathrm{mmol} / \mathrm{L}$ & $3.1(1.2-5.9)$ & $\uparrow 6.4(4.2-7.2)$ & 0.04 & $1.6(1.4-2.2)$ & 0.06 \\
\hline \multicolumn{6}{|l|}{ Treatments after birth, n\% } \\
\hline Antiviral therapy & $0(0.0)$ & $0(0.0)$ & NA & $0(0.0)$ & NA \\
\hline Antibiotic therapy & $3(23.1)$ & $5(45.5)$ & 0.39 & $1(8.3)$ & 0.59 \\
\hline Phototherapy & $5(38.5)$ & $1(9.1)$ & 0.17 & $4(33.3)$ & 1.00 \\
\hline Ventilation & $1(7.7)$ & $1(9.1)$ & 1.00 & $1(8.3)$ & 1.00 \\
\hline \multicolumn{6}{|l|}{ Outcomes, n\% } \\
\hline Taken home & $5(38.5)$ & $2(18.2)$ & 0.39 & $8(66.7)$ & 0.24 \\
\hline Hospitalization & $8(61.5)$ & $9(81.8)$ & 0.39 & $4(33.3)$ & 0.24 \\
\hline Death & $0(0.0)$ & $0(0.0)$ & NA & $0(0.0)$ & NA \\
\hline \multicolumn{6}{|c|}{ C-section = cesarean-section. IVF = in vitro fertilization. } \\
\hline \multicolumn{6}{|c|}{$\begin{array}{l}\text { Throat swab for SARS-CoV-2 virus were not done in all the neonates born to mother of the normal } \\
\text { group. }\end{array}$} \\
\hline \multicolumn{6}{|c|}{$\begin{array}{l}\otimes \text { Chest X-ray were not taken in } 5(38.5 \%), 3(27.3 \%), 3(25.0 \%) \text { neonates born to mothers of the } \\
\text { confirmed group, the clinical diagnosis group, and the normal group, respectively. }\end{array}$} \\
\hline \multicolumn{6}{|c|}{$\begin{array}{l}\text { Q Laboratory tests were not tested in } 5(38.5 \%), 1(9.1 \%), 8(66.7 \%) \text { neonates born to mothers of the } \\
\text { confirmed group, the clinical diagnosis group, and the normal group, respectively. }\end{array}$} \\
\hline
\end{tabular}


Though throat swab were taken for neonates born to mothers of the confirmed group and the suspected group, there were no positive results for the presence of SARS-CoV-2. Chest X-ray showed abnormalities in 2, 3, 2 neonates in the confirmed group, the suspected group, and the normal group, respectively. Four of the abnormalities were due to neonate respiratory distress syndrome, one was due to meconium aspiration syndrome, and two were of unknown causes.

For neonates who were in an unstable condition and required hospitalization, laboratory findings were measured within the first 24 hours after birth. Both the confirmed group and the suspected group had reduced red blood cells, but were not statistically significant with that of the normal group. Comparing to the confirmed group, the suspect group had elevated level of neutrophil count and total bilirubin, the normal group had an increased level of lymphocyte count; however, all the results were within normal ranges. The only abnormality, which had statistical significance, was the elevation of lactate in babies of the suspected group $(6.4 \mathrm{mmol} / \mathrm{L}, \mathrm{p}=0,04)$.

No infants received antiviral treatment, however, the antibiotic therapy, the phototherapy and the ventilation were implemented according to needs. These postnatal treatments were similar among three groups. The majority of the neonates born to mothers of the confirmed group and the suspected group were hospitalized $(61.5 \%$ vs. $81.8 \%, p=0.39)$. Two-thirds neonates born to the normal group were taken home directly after birth. None of the infants died.

\section{Comment}

We identified 11 pregnant women with laboratory-confirmed COVID-19, and included 11 aged paired suspect pregnant women and 11 normal women. All the women were residents of Wuhan; however, the majority of the confirmed women and the suspected women did not have any contact with patients who were suspected of COVID-19 during their third trimester. Only three women recalled that their family members, with whom they had close contact, had cough and fever as symptoms but none of them were diagnosed of COVID-19 infection. These women all stayed at home during their third trimester of pregnancy and only went to the hospital for the delivery. These suggest that the source of transmission is hard to identify and the vectors are likely to be asymptomatic, as reported by earlier report.[18]

The major early symptoms of 11 confirmed women were fever (45.5\%) and fatigue (45.5\%), and of the suspected women was fever $(72.7 \%)$. The peak temperature was mildly elevated, with median of $37.0^{\circ} \mathrm{C}$ and $37.6{ }^{\circ} \mathrm{C}$, which was very unspecific. Other signs and symptoms, such as dry cough, dyspnea, diarrhea, headache were uncommon manifestations of the pregnant women, making early consultation and the diagnosis from symptoms even harder. In fact, eight of eleven (72.7\%) pregnant women in the confirmed group were accidentally found to have radiologic abnormalities during the chest CT screening scan on admission for delivery. After the detection of abnormalities on chest CT scan, they were tested positive for RT-PCR during subsequent hospitalization. Otherwise, they felt nothing unusual. This highlighted the necessity of chest CT screening on admission in areas where the disease is prevalent. A majority (95.5\%) women of the confirmed and suspected groups had the radiologic abnormalities that ranged less than 
$25 \%$ of the lungs. Some patients of the confirmed group had atypical changes of the chest CT scans, such as bilateral pleural effusions and slightly increased densities. This further proofed the insidious onset of the disease and alerted the necessity of protection for healthcare workers.

Laboratory findings showed that the confirmed group and the suspected group had decreased level of lymphocyte, hemoglobin and albumin, and an increased level of lactate dehydrogenase, comparing to the normal group. The lymphocytopenia $\left(<1.5 \times 10^{9} / \mathrm{L}\right)$ is consistent with previous studies. $[4,6,19,20]$ In all the pregnant women, infected or not, the white blood cell count, neutrophil count and D-dimer were slightly increased. However, the level of platelet, prothrombin time, liver and renal function remained normal. None of the women needed mechanical ventilation, developed any complications, or died.

From the above, we concluded that the pregnant women of the confirmed and the suspected groups had milder symptoms and smaller chest CT abnormality ranges than non-pregnant adults infected with SARSCoV-2 and they also had a better outcome.[4] As we all know that pregnant women are at an immunosuppressive state, and this might turn out to be a double-edged sword. On the one hand, this lead pregnant women particularly susceptible to viral infection. On the other hand, this state might prevent injuries from overactivated cytokines storms that consequently lead to acute respiratory distress syndrome (ARDS) and hypoxemia. This might explain why the infected pregnant women were often absent of symptoms and were less likely to develop severe COVID-19 pneumonia. We thus recommend chest CT screening with necessary abdominal protection for pregnant women on admission in outbreak areas, due to the high sensitivity and time efficiency of CT scan and the difficulty of diagnosis in pregnant women.[21]

Though some medications seemed to reduce viral load significantly, the efficacy and safety in vivo still need further evaluation before clinical treatment.[22-24] The pregnant women thus did not receive any treatment before the delivery and were treated with antibiotics after the delivery, especially after the Csection operation. Women in the confirmed group and the suspected group also had oxygen support and interferon inhalation and antiviral therapy. However, the outcomes were not different.

For neonates born to mothers with confirmed COVID-19, there is no evidence of premature birth, low birth weight, or complications associated with the disease. Only one neonate had a 1-min Apgar score of 7 and all had 5-min Apgar score of 8-10. There were no stillbirth or death. Some infants had fever, dyspnea, lethargy, moaning, feeding intolerance, and cyanosis, however, there was no statistical significance among three groups. We thus conclude that the COVID-19 of mothers do not have significant influences on their babies. No infants were tested positive for SARS-CoV-2 and intrauterine fetal infection was unlikely which is in accordance with previous studies. $[25,26]$

To our knowledge, this is the first comparison cohort study that compared pregnant women with confirmed COVID-19 infection to age-matched pregnant women with suspected infectious and to agematched healthy pregnant women. Unlike previous studies which concluded that clinical symtoms of COVID-119 in pregnant women were not different from those of the general population, our study found 
that the onset of the disease was asymptomatic and insidious in pregnanct women.[25,26] Our study also found that the infection of COVID-19 did not increase the risk for premature delivery, premature rupture of membrane, or comorbidities in pregnancy. Our study has several limitations. First, the sample size is small, because laboratory-confirmed women were hard to identify. Further study with larger sample size needs to be investigated to improve results reliability and to reduce selection bias. Second, all the women included were in their third trimester of pregnancy. Whether the infection of COVID-19 during early pregnancy trimesters will have effects on the fetus still need to be clarified. Third, the exposure history of suspicious patients was unreliable due to recall bias. Fourth, we did not collect samples of amniotic fluid, cord blood, vaginal mucosa or shedding in birth canals to ascertain the possibility of vertical transmission. Fifth, the test of IgM and IgG as specific antibodies for the SARS-CoV-2 were not brought forward at the time of the study inclusion. Future studies with up-to-date diagnosis methods and treatments could be investigated, and long-term follow-up of postpartum women and children need to be studied.

\section{Conclusions}

In comparison to the general population, pregnant women had insidious onset of the COVID-19 and were often asymptomatic. Chest CT scan is important for screening on hospital admission in disease prevalent areas. COVID-19 infection during third trimester of pregnancy did not seem to increase the risk of complications of pregnant women or have impacts on their babies.

\section{Declarations}

\section{Acknowledgement}

This work was supported by the National Natural Science Foundation of China (No. 81971816; No. 81772046)

\section{Availability of the data and materials}

All included data are available in the public domain, and all references are included in our reference list. Extracted data and calculations will be made available to individual scientists upon reasonable request.

\section{Contributions:}

Conceived the project, designed the project, extract and analyzed data, drafted the manuscript and approved the final manuscript: Q.X.

Conceived the project, extract the data: Q. G.

Revise the manuscript and approved the final manuscript: 
Data collection: J. H., D.Z., S. C., L. S., Y. L.

Designed the project, revised the manuscript and approved the final version: Z.P, Y. L.

\section{Ethics declarations}

\section{Ethics approval and consent to participate}

This study was reviewed and approved by the institutional ethics board of Zhongnan Hospital of Wuhan University (No. 2020020) and Women and Children's Hospital of Hubei Province(NO. LW035). We have obtained both informed consent and ethics committee approval for studies on patients, patient records, or volunteers.

\section{Consent for publication}

All scientists named in the acknowledgments section below consented to be acknowledged in this publication. We did not include any other individual person's data in any form.

\section{Disclosure:}

The authors declare no competing interests.

\section{References}

1. World Health Organization. Naming the coronavirus disease (COVID-19) and the virus that causes it. Accessed February 28. 2020. https://www.who.int/emergencies/diseases/novel-coronavirus2019/technical-guidance/naming-the-coronavirus-disease-(covid-2019)-and-the-virus-that-causes-it.

2. Hui DS, Madani EIA. TA et al: The continuing 2019-nCoV epidemic threat of novel coronaviruses to global health - The latest 2019 novel coronavirus outbreak in Wuhan, China. Int J Infect Dis. 2020;91:264-6.

3. Liangos $\mathrm{O}$, Wald R, O'Bell JW, et al. Epidemiology and outcomes of acute renal failure in hospitalized patients: a national survey. Clin J Am Soc Nephrol. 2006;1(1):43-51.

4. Wang D, Hu B, Hu C, et al: Clinical Characteristics of 138 Hospitalized Patients With 2019 Novel Coronavirus-Infected Pneumonia in Wuhan, China. JAMA 2020.

5. Lu H, Stratton CW, Tang YW. Outbreak of pneumonia of unknown etiology in Wuhan, China: The mystery and the miracle. J Med Virol. 2020;92(4):401-2.

6. Chen N, Zhou M, Dong X, et al. Epidemiological and clinical characteristics of 99 cases of 2019 novel coronavirus pneumonia in Wuhan, China: a descriptive study. Lancet. 2020;395(10223):507-13.

7. Mosser DM, Edwards JP. Exploring the full spectrum of macrophage activation. Nat Rev Immunol. 2008;8(12):958-69. 
8. Coronavirus Update (Live): 935,957 Cases and 47,245 Deaths from COVID-19 Virus OutbreakWorldometer. Retrieved 2 April 2020. https://www.worldometers.info/coronavirus/.

9. Wald R, Quinn RR, Luo J, et al. Chronic dialysis and death among survivors of acute kidney injury requiring dialysis. JAMA. 2009;302(11):1179-85.

10. Novak ML, Koh TJ. Macrophage phenotypes during tissue repair. J Leukoc Biol. 2013;93(6):875-81.

11. Centers for Disease Control and Prevention. Coronavirus Disease 2019 (COVID-19) Symptoms. 10 February 2020. https://www.cdc.gov/coronavirus/2019-ncov/about/symptoms.html.

12. World Health Organization (WHO). Q\&A on coronaviruses (COVID-19). Accessed on 20 January, 2020. https://www.who.int/news-room/q-a-detail/q-a-coronaviruses.

13. Jang HS, Kim J, Park YK, et al. Infiltrated macrophages contribute to recovery after ischemic injury but not to ischemic preconditioning in kidneys. Transplantation. 2008;85(3):447-55.

14. National Health Commission of China. New coronavirus pneumonia prevention and control program (5th edn). Feb 21. 2020.

http://www.nhc.gov.cn/jkj/s3578/202002/dc7f3a7326e249c0bad0155960094b0b.shtml.

15. WHO. Clinical management of severe acute respiratory infection when novel coronavirus (nCoV) infection is suspected. Interim guidance. Jan 12. 2020. https://www. who.int/docs/default-source/ coronaviruse/clinical-management-of-novel-cov.pdf (accessed Feb 4, 2020).

16. Zhou D, Huang C, Lin Z, et al. Macrophage polarization and function with emphasis on the evolving roles of coordinated regulation of cellular signaling pathways. Cell Signal. 2014;26(2):192-7.

17. Zarjou A, Agarwal A. Sepsis and acute kidney injury. J Am Soc Nephrol. 2011;22(6):999-1006.

18. Rothe $C$, Schunk M, Sothmann P, et al. Transmission of 2019-nCoV Infection from an Asymptomatic Contact in Germany. N Engl J Med. 2020;382(10):970-1.

19. Guan WJ, Ni ZY, Hu Y, et al: Clinical Characteristics of Coronavirus Disease 2019 in China. N Eng/ J Med 2020.

20. Huang C, Wang Y, Li X, et al. Clinical features of patients infected with 2019 novel coronavirus in Wuhan, China. Lancet. 2020;395(10223):497-506.

21. Ai T, Yang Z, Hou H, et al: Correlation of Chest CT and RT-PCR Testing in Coronavirus Disease 2019 (COVID-19) in China: A Report of 1014 Cases. Radiology 2020:200642.

22. Wang $M$, Cao $R$, Zhang $L$, et al. Remdesivir and chloroquine effectively inhibit the recently emerged novel coronavirus (2019-nCoV) in vitro. Cell Res. 2020;30(3):269-71.

23. Lim J, Jeon S, Shin HY, et al. Case of the Index Patient Who Caused Tertiary Transmission of COVID19 Infection in Korea: the Application of Lopinavir/Ritonavir for the Treatment of COVID-19 Infected Pneumonia Monitored by Quantitative RT-PCR. J Korean Med Sci. 2020;35(6):e79.

24. Gautret P, Lagier JC, Parola P, et al: Hydroxychloroquine and azithromycin as a treatment of COVID19: results of an open-label non-randomized clinical trial. Int J Antimicrob Agents 2020:105949.

25. Huijun Chen JG, Chen W, Luo F, Yu X, Zhang W, Li J, Zhao D, Xu D, Gong Q, Liao J, HUixia Yang, Wei Hou, Yuanzhen Zhang: Clinical characteristics and intrauterine vertical transmission potential of 
COVID-19 infection in nine pregnant women: a retrospective review of medical records. The Lancet 2020.

26. Huaping Zhu LW, Chengzhi F, Peng S, Zhang L. Guiping Chang, Shiwen Xia: Clinical analysis of 10 neonates born to mothers with 2019-nCov pneumonia. Translational Pediatrics 2020.

\section{Figures}
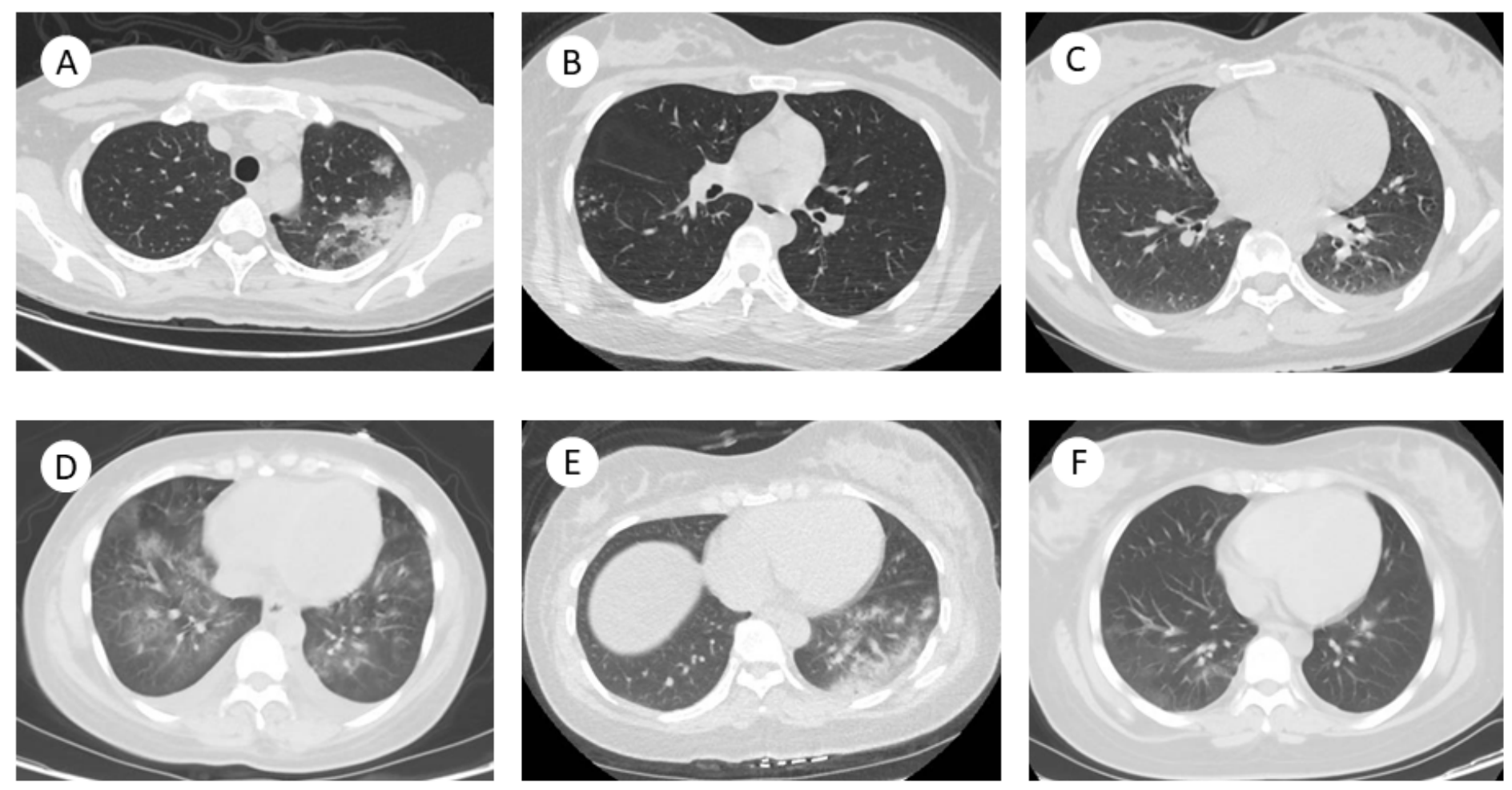

Figure 1

Chest CT scans (transverse plan) of six patients A-C: pregnant women in the confirmed group. (A) Patient 1: multiple bilateral ground-glass opacities and left-sided patchy consolidation. (B) Patient 2: increased density in the left lower lobe. (C) Patient 3: left-sided pleural effusion. D-F: pregnant women in the suspected group. (D) Patient 4: multiple bilateral ground-glass opacities, bilateral pleural effusion. (E) Patient 5: multiple bilateral ground-glass opacities, prominent on the left. (F) Patient 6: multiple bilateral ground-glass opacities, prominent on the right. 